\title{
Patterns at the Onset of Electroconvection in Freely Suspended Smectic Films
}

\author{
Stephen W. Morris, ${ }^{1}$ John R. de Bruyn, ${ }^{2}$ and A. D. May ${ }^{3}$
}

\begin{abstract}
We report the results of experiments on electrically driven convection that occurs in a thin, freely suspended film of smectic A liquid crystal when an electric field is applied in the plane of the film. Convection in a vortex pattern is found above a well-defined critical voltage. The film behaves as a twodimensional isotropic liquid: neither its thickness nor the director field are modified by the flow. We present measurements of the critical voltage at the onset of convection in two experimental configurations- one which allows the injection of charges into the film from the electrodes, and one which does not. When injection is present, the critical voltage for the onset of flow increases monotonically with increasing frequency of applied field. With no injection, there is no instability at $\mathrm{DC}$ and the critical voltage diverges there. The nature of the flow pattern observed at onset changes with frequency. Below a certain frequency the film flows in vortices that extend over the width of the film; above this frequency the flow is confined to two lines of smaller vortices localized along the electrodes. We present a simple discussion of the mechanisms which drive the convection.
\end{abstract}

KEY WORDS: Convection; pattern formation; smectic liquid crystals; twodimensional liquid; electroconvection.

\section{INTRODUCTION}

Patterns are common in nonlinear dissipative systems, both in nature and in the laboratory. Typically a pattern will first develop when such a system

\footnotetext{
${ }^{1}$ Department of Physics, University of Toronto, Toronto, Ontario, Canada M5S 1A7. Present address: Department of Physics, University of California, Santa Barbara, California 931069530.

${ }^{2}$ Department of Physics, Memorial University of Newfoundland, St. John's, Newfoundland, Canada A1B 3X7.

${ }^{3}$ Department of Physics and Ontario Laser and Lightwave Research Center, University of Toronto, Toronto, Ontario, Canada M5S 1A7.
} 
undergoes a bifurcation from a uniform, time-independent state to one with some form of organized spatial or temporal structure. In the study of the formation, stability, and evolution of patterns, it is simplest to consider experimental situations where the first bifurcation is to a simple one-dimensional pattern. ${ }^{(1)}$ This approach has led to detailed investigations of, for example, Rayleigh-Bénard convection, ${ }^{(1,2)}$ Taylor vortex flow, ${ }^{(1,3)}$ and electroconvection in nematic liquid crystals. ${ }^{(4)}$ Some very recent work on these systems is discussed elsewhere in these proceedings. ${ }^{4}$ Secondary instabilities occur in all these systems when they are driven further from equilibrium, ${ }^{(2-5)}$ which leads to complex, often time-varying, threedimensional flow and eventually to turbulence.

We report here on studies of the patterns which develop when a thin film of smectic A liquid crystal with two free surfaces is driven out of equilibrium by the application of an electric field in the plane of the film. Above a critical applied voltage we observe a bifurcation from a state with no flow to one in which the film flows in a linear pattern of vortices. The critical voltage and the wavelength of the pattern are dependent on the frequency of the applied field. The structure of the smectic, discussed below, constrains the film to behave like a two-dimensional fluid and threedimensional instabilities are strongly inhibited. The mechanism leading to the initial instability of the rest state does not require the anisotropy of the liquid, in contrast to that usually studied in the electroconvection of nematics. ${ }^{(4,5-9)}$ Preliminary reports of this work have appeared elsewhere. ${ }^{(10,11)}$

The liquid crystal used was $8 \mathrm{CB}\left(4,4^{\prime}-n\right.$-octylcyanobiphenyl), which has a smectic A phase between 21 and $33.5^{\circ} \mathrm{C}$; our experiments were performed at room temperature $\left(23^{\circ} \mathrm{C}\right)$. The rodlike liquid crystal molecules have an orientational order which is characterized by the director, ${ }^{(6,7)}$ a vector pointing in the mean direction of the long molecular axis. In addition to their orientational order, the molecules in the smectic A phase are positionally ordered into layers with the director aligned normal to the layer planes. In $8 \mathrm{CB}$ these layers have a thickness of $3.16 \mathrm{~nm}$, which is about 1.4 molecular lengths. ${ }^{(12)}$ The smectic A phase has very strongly anisotropic flow properties. For shears in the plane of the layers, it behaves as a liquid with a viscosity of order 1 Poise. ${ }^{(13,14)}$ In the perpendicular direction it is similar to a plastic crystal. When the orientation of the layers is fixed by external boundaries, flow perpendicular to the layers is possible only by the "permeation" effect, ${ }^{(6,7,13)}$ in which molecules

\footnotetext{
${ }^{4}$ See the papers in these proceedings by Cannell et al., Surko et al., Ciliberto et al., (RayleighBénard convection), Tagg et al., Weiner et al. (Taylor vortex flow), Rehberg et al., Kramer et al., and Ribotta et al. (electroconvection in liquid crystals) for reports of recent work on pattern formation in these systems.
} 
move from layer to layer. This type of flow has been observed in $8 \mathrm{CB},{ }^{(13)}$ and is completely negligible at the temperature used in our experiment, which is far from the smectic A to nematic phase transition. If the layers are not fixed, then perpendicular shears cause the formation of defects in the layer structure and singular lines in the director field. ${ }^{(13)}$ In the smectic A phase, distortions of the director field are always accompanied by distortions of the smectic layers. ${ }^{(6,7)}$

In our experiments the convection took place in a thin film of smectic A which was supported only at its edges, rather like a soap film. In this configuration, smectics form very stable films which are an integer number of smectic layers thick. ${ }^{(15,16)}$ The thermodynamics and structure of static, freely suspended films have been extensively studied because of the general interest in systems of reduced dimensionality. ${ }^{(16)}$ Our experiment makes use of the unique flow properties and boundary conditions of these films. Their structure fixes the plane of the smectic layers and therefore suppresses any flow normal to the film. Flow-induced distortions of the director field are similarly suppressed. The free surfaces of the film have free-slip boundary conditions, so the film can be realistically treated as an isotropic, twodimensional liquid.

The dielectric anisotropy of $8 \mathrm{CB}$ is $\Delta \varepsilon=\varepsilon_{||}-\varepsilon_{\perp} \approx 8.5$, where $\varepsilon_{||}$and $\varepsilon_{\perp}$ are the dielectric constants parallel and perpendicular to the director, respectively. ${ }^{(17)}$ Electroconvention of the Williams domain type, which is observed in certain nematics, is driven by the Carr-Helfrich ${ }^{(4-9)}$ mechanism. This mechanism is usually found in materials with a negative dielectric anisotropy and necessarily involves a reorientation of the director. The convective flow studied here involves no such reorientation and so cannot be attributed to this mechanism. As we discuss below, the mechanism of our convection also occurs in isotropic fluids; the anisotropy of the smectic only serves to force the system to be two-dimensional.

Electrically driven convection has been studied in suspended nematic and isotropic films by Faetti et al. ${ }^{(18-20)}$ They observed two types of behavior. The first they called the "domain mode"; this was observed in the nematic phase and disappeared at the nematic to isotropic phase transition. ${ }^{(19)}$ It exhibited behavior consistent with its being the twodimensional analog of Williams domains, driven by the Carr-Helfrich mechanism. They also observed a pattern which they referred to as the "vortex mode." ${ }^{20)}$ This state existed in both the nematic and isotropic phases, and must therefore be driven by a mechanism which does not require anisotropy. This second state is probably closely related to the phenomena observed by us in smectic films, although there are important differences which will be discussed in Section 4 below.

There is a large literature concerning electrically driven convection in 
bulk slabs of isotropic, dielectric liquids, ${ }^{(7,21-27)}$ which is also relevant to liquid crystals. In general, the instability arises due to the interaction of an applied field with space charge in the liquid. This space charge may originate from any of several processes. Charges of one or both signs may be injected into an otherwise perfectly insulating liquid from special electrodes. This mechanism has been extensively studied both theoretically ${ }^{(21-26)}$ and experimentally. ${ }^{(24-26)}$ However, the injection process can be treated theoretically only in a rather idealized way, and in practice liquid crystals are too impure to be regarded as insulators. An isotropic system which is more relevant to liquid crystals is an electrochemical cell ${ }^{(27,28)}$ in which the fluid is an electrolyte with a nonzero conductivity. In this case the source of space charge is electrochemical reactions at the electrodes, possibly accompanied by reactions in the bulk.

Under AC driving it is possible to achieve a transient unstable charge distribution and transient convection in liquids which are not perfectly insulating. ${ }^{(7,29,30)}$ In this process there is no net transfer of charge through the circuit, so neither injection nor electrochemical reactions are required as a source of charges; only the transient rearrangement of charge carriers already in the liquid is involved. To our knowledge, this process has never been fully treated theoretically. ${ }^{(29,30)}$ Our observations suggest that both the injection and the transient processes contribute to the convection behavior of the smectic film.

The remainder of this paper is organized as follows: In Section 2 we describe our experiment and experimental procedures. We present results of measurements of the flow pattern close to its onset in Section 3. We outline the mechanism responsible for the observed behavior and compare our results to previous work in Section 4, which is followed by a brief summary.

\section{EXPERIMENT}

The experiments were performed on a rectangular film of smectic A liquid crystal suspended on a holder which supported the film's edges but left its top and bottom surfaces free. The two types of holder used are shown in Fig. 1. The first consisted of two parallel $15-\mu$ m-diameter tungsten wire electrodes which supported the long edges of the film. The ends were supported by wipers made from coverslip glass. One of the wipers could be moved in the direction parallel to the electrodes by a micropositioning motor drive. In this configuration, charged species could be injected into the film via electrochemical reactions at the electrodes, which were in direct contact with the film. We refer to this as the contact holder. 

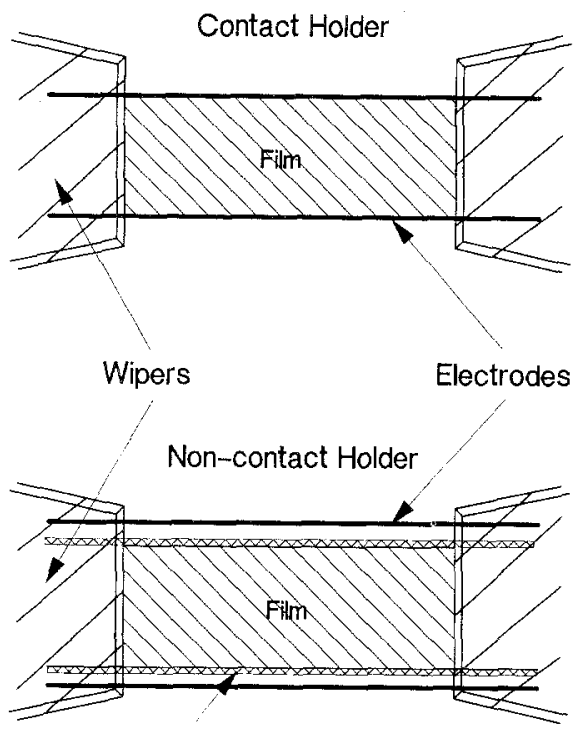

Nylon Threads

Fig. 1. The two types of film holder used. In the contact holder the electrodes are in direct contact with the liquid, while in the noncontact holder the DC current path is blocked by an air gap (not shown to scale).

The second holder was similar, except that the long sides of the film were supported by $125-\mu \mathrm{m}$ nylon threads. Tungsten wire electrodes lay outside of these threads, separated from them by a $125-\mu \mathrm{m}$ air gap. The ends of the film were again supported by glass wipers. In this arrangement, the electrodes were not in contact with the film and injection of charges from the electrodes could not occur. This is referred to as the noncontact holder.

In both holders the width $d$ of the film was mechanically adjustable from 0 to $3 \mathrm{~mm}$. One electrode was held at ground potential and a bipolar voltage $V(t)$ was applied to the other using a computer-controlled function generator. We found that the symmetry of the flow pattern which formed in the convecting film was sensitive to the symmetry of the field near the electrodes. If the holders were placed in a grounded enclosure, the asymmetry of the field caused the flow pattern to form nearer to the ungrounded electrode. To avoid this, we made the field as symmetric as possible with respect to the two electrodes by surrounding them with guard electrodes biased at $V(t) / 2$. Thus the wires had potentials of $\pm V(t) / 2$ with respect to their enclosure. The film holder and guard electrodes were contained inside 
an aluminum housing which provided electrical, mechanical, and thermal shielding.

The conductivity of liquid crystals is dominated by ionized impurities ${ }^{(7)}$ whose species are often unknown in normal commercial materials. The convective flow studied here is observed at relatively low frequencies, under which conditions the electrochemical behavior of the impurities is important. In particular, it is important that their electrochemical reactions be reversible to prevent sample degradation during the experiment. ${ }^{(7,31)}$ In order to control these effects, we doped our $8 \mathrm{CB}$ as received from the manufacturer (BDH Ltd, Poole, England) with $7.5 \pm 0.2 \mathrm{mmol} /$ liter of TCNQ (tetracyanoquinodimethane). TCNQ is an electron acceptor which when used as a dopant in nematics is believed to form charge transfer complexes ${ }^{(32)}$ with the liquid crystal molecules and has been shown to give strong reversible injection of $\mathrm{TCNQ}^{-}$ions at the cathode. $^{(31)}$

To draw a film, the two glass wipers were brought together. A small amount of $8 \mathrm{CB}$ was placed at the join between the wipers, which were then separated at an adjustable rate in the range $1-10 \mu \mathrm{m} / \mathrm{sec}$ using the motor drive. The motorized wiper was stopped when the film reached the desired length $L$. For the work reported here, the films had a width $d \approx 2 \mathrm{~mm}$ and an aspect ratio $L / d$ greater than 10 .

Our films were characterized and the flow visualized using simple optical techniques. The films used contained between 20 and a few hundred smectic layers, corresponding to thickness in the range $0.060 .8 \mu \mathrm{m}$. The films therefore had thicknesses comparable to the wavelength of visible light. When viewed in a low-power microscope in reflected white light, a film appears brightly colored as a result of the interference between light reflected from its top and bottom surfaces. ${ }^{(16)}$ Unlike a conventional soap film, a smectic film of nonuniform thickness shows discontinuous changes in the pattern of reflected colors due to steps in the thickness forced by the requirement that the film be an integer number of smectic layers thick. The uniformity of the film thickness could therefore be checked simply by observing the uniformity of the film color.

The variations in color across a nonuniform film allowed for a simple method of flow visualization. Convection in such a film causes a spectacular swirling of the colors as the thickness variations are advected with the flow. This can be easily followed under the microscope either by eye or on a video monitor. Pictures recorded using nonuniform films are very beautiful to watch, but provide only qualitative information about the flow pattern because of the thickness dependence of the critical voltage. For quantitative measurements, only films of completely uniform thickness are used. In such films we find that the flow disturbs neither the thickness nor 
the director orientation, so that the flow cannot be observed using the optical properties of the liquid itself. In order to study the flow, we introduced a small amount of dust into the air in the experimental apparatus, some of which settled on the film. When the film is illuminated from behind with an expanded beam of a laser, the light scattered by the dust is visible as bright specks which move with the flow in the film as illustrated in Fig. 2. Our results are not affected by the amount or type of dust used, within reasonable limits.

The actual thickness of a uniform film was found by measuring its reflectivity. The reflectivity at a fixed wavelength $\lambda$ for light polarized perpendicular to the plane of incidence is a periodic function of the thickness with period $\lambda / 2 n_{\perp}$ with maxima at odd multiples of $\lambda / 4 n_{\perp} \cdot{ }^{(33,34)}$ Here $n_{\perp}$ is the refractive index perpendicular to the director. Thus, the thickness can be unambiguously determined from the reflectivity at a single wavelength only if the film is thinner than $\lambda / 4 n_{\perp}$, which corresponds to about 30 smectic layers for $8 \mathrm{CB}$. This method has been widely used in studies of thin static films. ${ }^{(34)}$ However, we have found it difficult to study convection in films this thin because the flow tended to draw excess material off the edges to form thicker islands in the film. We therefore simply measure the reflectivity of our thicker films at several incommensurate wavelengths and fit the reflectivity as a function of wavelength. We can thus determine the thickness of films up to several hundred layers thick. A $10-\mathrm{mW} \mathrm{Ar}^{+}$laser polarized perpendicular to the plane of incidence was reflected off the film and the reflectivity was measured at each of the six wavelengths available, which ranged from 457.9 to $514.5 \mathrm{~nm}$. We make use of the known refractive index ${ }^{(17)}$ and layer spacing ${ }^{(12)}$ of $8 \mathrm{CB}$ to determine the number of smectic layers to the nearest integer. For films less

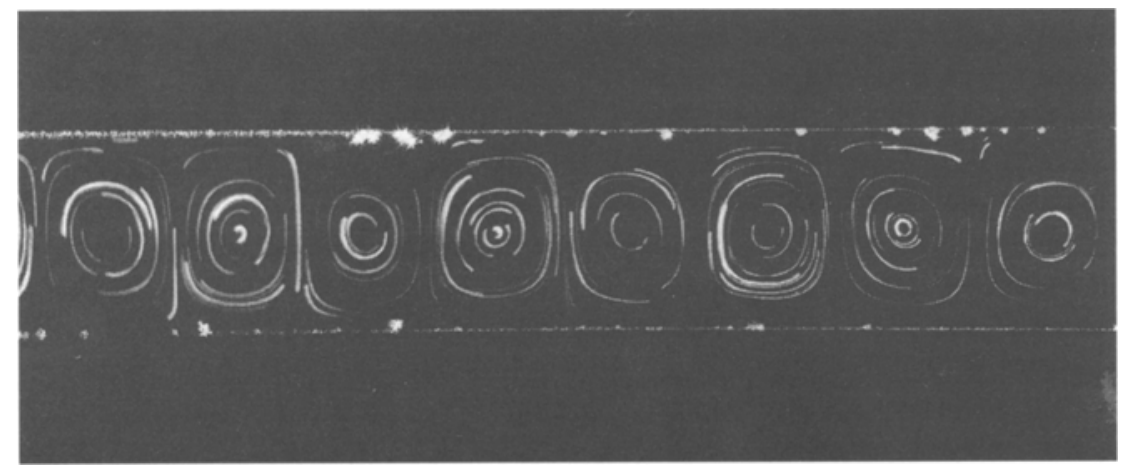

Fig. 2. The vortex pattern at DC in the contact holder. The film was $2 \mathrm{~mm}$ across. Only a portion of the length of the film is shown. 
than 100 layers thick, a single layer change represents a considerable change in reflectivity, so that one can easily find the thickness to \pm 1 layer. For thicker films, which appear nearly white in reflected light, the uncertainty rises to \pm 2 layers, and a small loss correction must be included in the calculation. ${ }^{(33)}$

\section{RESULTS}

When a DC voltage is applied to the contact holder, there is a welldefined critical voltage above which a flow pattern develops in the film. The film convects in a stationary one-dimensional pattern of vortices with alternating vorticity, as shown in Fig. 2. As the applied voltage is increased, the flow velocity increases, but the vortex pattern remains steady until, at about 10 times the critical voltage, the flow abruptly becomes unsteady. In this paper, we confine our attention to patterns near the onset of convection in films of various thicknesses as a function of applied voltage and frequency.

We find that the uniform thickness of the film is undisturbed by the flow. This was determined by observing the uniformity of the film color as described above, and by measuring the film thickness before and after an experimental run. In addition, the flow is not accompanied by any distortion of the director field. Such a distortion would change the local optic axis of the film and would thus be observable when the film is viewed between crossed polarizers; no such effect was observed in any of our experiments. These results differ markedly from those found in nematic films by Faetti et al., ${ }^{(18-20)}$ as will be discussed below.

When the contact holder is driven with a time-varying voltage $V(t)$, the film convects whenever $|V(t)|$ exceeds a critical value $V_{c}(f)$, where $f$ is the frequency. The direction of rotation of the vortices reverses every halfperiod as the field direction reverses. With a low-enough-frequency sinewave driving, the flow has time to die out due to viscous damping during the time that $|V(t)|<V_{c}(f)$. In this case, the position of the vortices may differ from one-period to the next. $V_{c}(f)$ for a typical film is shown in Fig. 3. The onset was determined by watching the motion of the visualization particles through the microscope or on a video monitor. Near $V_{c}(f)$ there is generally some disorganized motion of the film. Vortexlike flow first appears at the ends of the film, presumably due to end effects which modify the electric field there. We define $V_{c}(f)$ as the amplitude of the applied voltage at which the flow becomes organized into vortices throughout the entire length of the film. Within the uncertainty in our visual determination of the onset, we observe no hysteresis in $V_{c}(f)$, that 


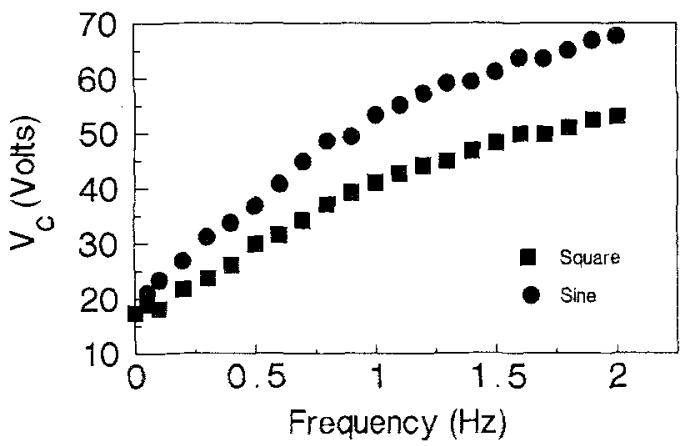

Fig. 3. The critical voltage versus frequency for the contact holder.

is, the onset of convection appears to be a forward bifurcation which is slightly imperfect due to the end effects.

It is interesting to compare the response of the film in the contact holder to sinusoidal and square wave voltages. For square waves, the flow is steady during the constant part of the waveform, but executes a sudden reversal of direction at each zero crossing. For sine waves, the flow reverses more smoothly. $V_{c}(f)$ for both waveforms is shown in Fig. 3 and the ratio $V_{c}^{\text {sine }} / V_{c}^{\text {square }}$ is shown in Fig. 4 . This ratio is approximately $4 / \pi$ for frequencies above about $0.2 \mathrm{~Hz}$ for films of all thicknesses studied. This is equal to the ratio of the first Fourier components of the two waveforms. Below $0.2 \mathrm{~Hz}$ the ratio goes to 1 as the values of $V_{c}(f)$ approach a common DC value.

At a given frequency, $V_{c}(f)$ is linear in the thickness $s$ over the range of thickness and frequencies studied. This is shown in Fig. 5, for two

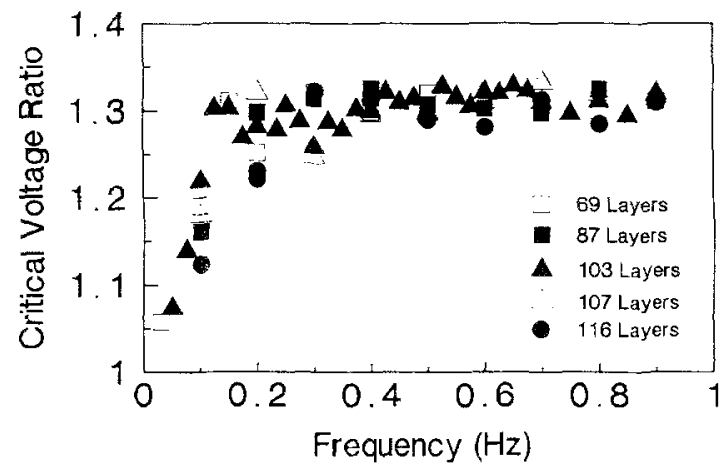

Fig. 4. The ratio of the critical voltages for sine and square waves in the contact holder, versus frequency. 


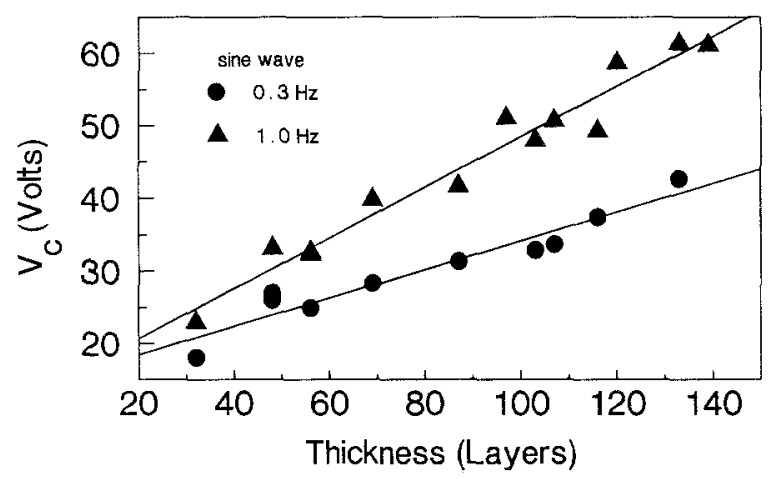

Fig. 5. The critical voltage versus thickness in layers in the contact holder, for two fixed frequencies.

frequencies. The slopes of these lines, $\partial V_{c} /\left.\partial s\right|_{f}$, increase linearly with frequency as shown in Fig. 6.

In the noncontact holder the flow is somewhat different in nature. The most important observation is simply that convection does occur in the noncontact holder at nonzero frequency, with a flow pattern similar to that in the contact holder. No flow is observed at DC, and, with an AC driving voltage, the flow first occurs at the zero crossings of the applied voltage, where the field is most rapidly changing, and not at the peaks as in the contact holder. $V_{c}(f)$ for the noncontact holder is shown in Fig. 7. $V_{c}$ for sinusoidal voltages diverges at low frequency. Under a low-frequency square-wave voltage, the film does not convect during the constant part of the waveform, but exhibits transient bursts of convection at the zero

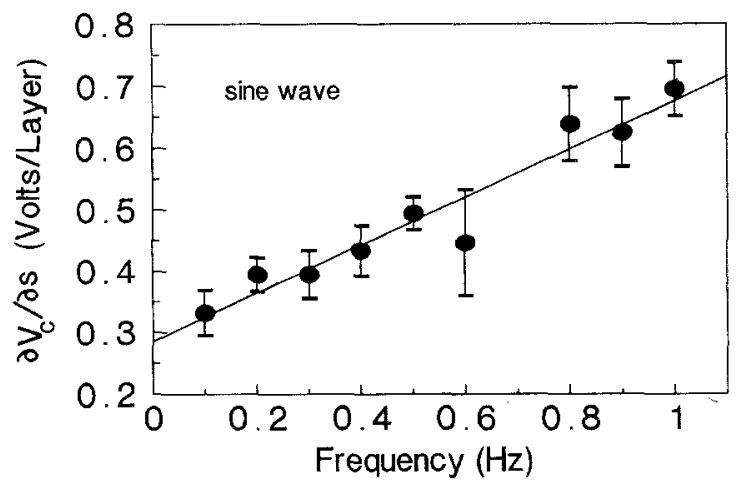

Fig. 6. The rate of change of critical voltage with thickness, at fixed frequency $\left.\left(\partial V_{c} / \partial s\right)\right|_{f}$, as a function of frequency. 


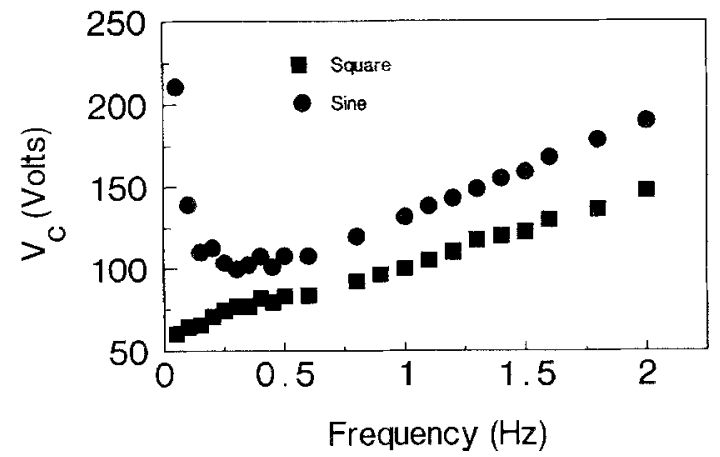

Fig. 7. The critical voltage versus frequency for the noncontact holder.

crossings. $V_{c}$ for square waves does not diverge at $\mathrm{DC}$, but approaches a constant value as $f \rightarrow 0$.

At low frequencies, the wavelength $\lambda$ of the pattern is nearly independent of voltage and frequency and proportional to the width of the film $d$ as shown in Fig. 8. We find $\lambda / d=1.30 \pm 0.05$ for a film in the contact holder at DC over the range $1<d<3 \mathrm{~mm}$. However, at frequencies above a few $\mathrm{Hz}$ the flow pattern which develops at onset changes: Figure 9 shows the pattern which appears at higher frequency. The roughly square vortices seen at low frequency are replaced by two lines of smaller vortices, one along each electrode. Figure 10 shows the reduced wavelength $\lambda / d$, at onset, as a function of frequency. The new pattern emerges continuously out of the old: the low-frequency vortices begin to narrow at about $2 \mathrm{~Hz}$ and the flow reorganizes into the smaller vortices. The pattern of small vortices

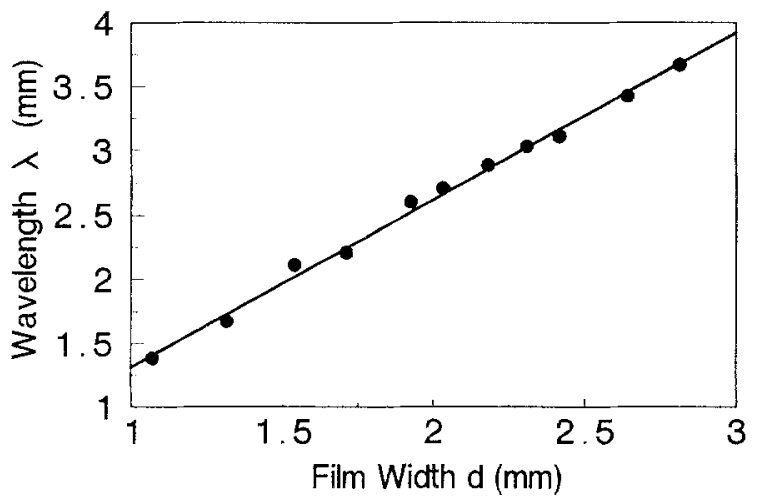

Fig. 8. The wavelength of the vortex pattern at DC versus the width of the film. 

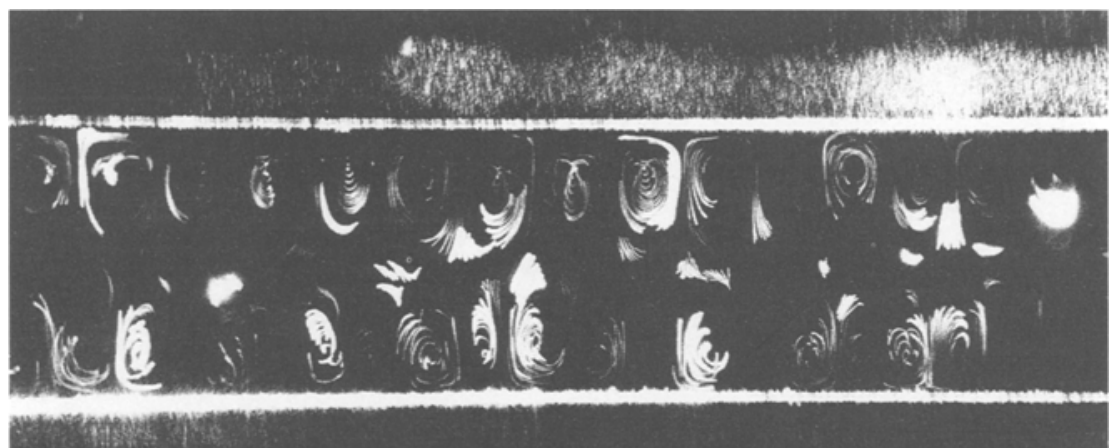

Fig. 9. The higher frequency flow pattern in the contact holder.

does not persist as the voltage is increased above $V_{c}(f)$; rather, the small vortices grow and coalesce until a pattern similar to the low-frequency vortices emerges. Figure 11 shows how $\lambda / d$ evolves as the voltage is increased beyond the onset of convection at $f=4 \mathrm{~Hz}$. The low-frequency value of $\lambda / d$ is restored at higher voltages, although the pattern is much less steady than near DC.

Our method of flow visualization works poorly for very small vortices for which there may only be one dust particle in a given vortex. This accounts for the scatter in the wavelength data at high frequency in Figs. 10 and 11. By watching the colors in nonuniform films, it is possible to follow the small vortices up to frequencies of about $50 \mathrm{~Hz}$. The wavelength of the vortices seems to decrease with frequency until there is flow only

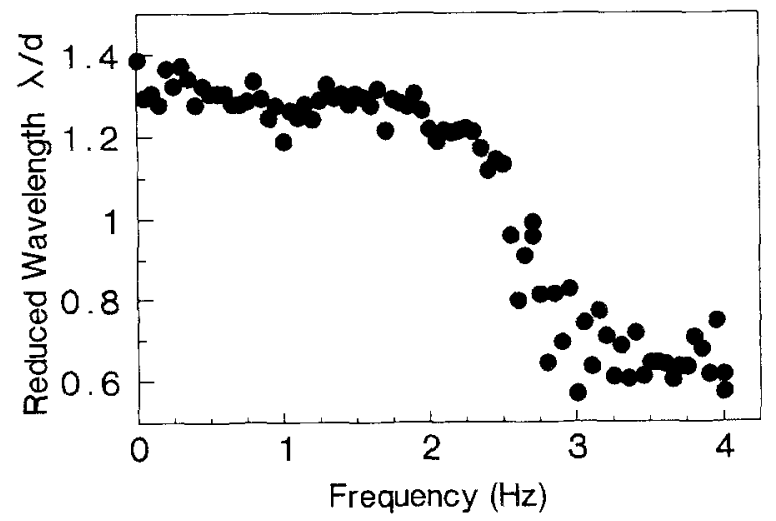

Fig. 10. The reduced wavelength $\lambda / d$ at onset, versus frequency. 


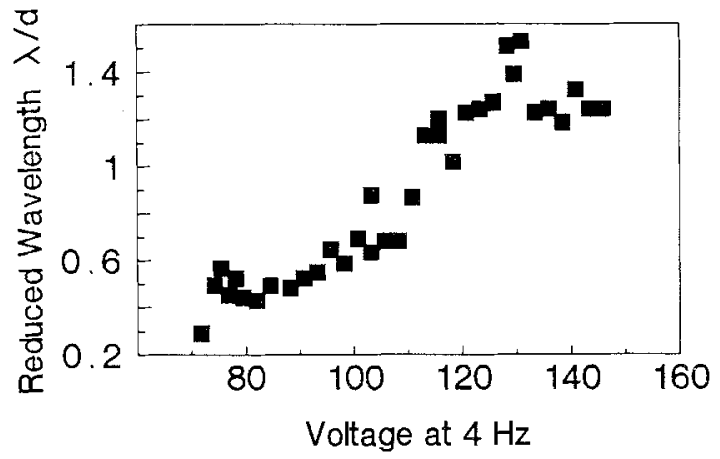

Fig. 11. The reduced wavelength $/ / d$ versus voltage, at a fixed frequency of $4 \mathrm{~Hz}$.

very close to each electrode. The behavior of the wavelength in the noncontact holder is similar to that in the contact holder.

We have also performed some qualitative experiments in an annular film holder. This holder was a machined annular slot with knife edges, having an inside diameter of $6 \mathrm{~mm}$ and an outside diameter of $12 \mathrm{~mm}$. The inside and outside edges were insulated from each another and the outside one was grounded while DC was applied to the inner one. Thus the film was driven with a radial electric field. This geometry is interesting because end effects are eliminated, and because radial driving fields are difficult to obtain in most convection experiments. Fourteen vortices are observed around the circumference of the annulus. Taking the length of the system to be the mean circumference and $d$ to be the width of the gap, we find $\lambda / d=1.35 \pm 0.05$, which is equal, within error, to the low-frequency value of the wavelength in the rectangular holders. The high-frequency pattern is also qualitatively similar to that seen in the rectangular holders; it consists of many small vortices localized around the inner electrode.

\section{DISCUSSION}

In any electroconvecting system, the onset of flow occurs when the force exerted by the electric field on the charges in the fluid can no longer be balanced by viscous effects, and the rest state of the fluid becomes unstable. To understand the instability mechanism completely, one must solve a system of coupled partial differential equations consisting of the Navier-Stokes equation,

$$
\frac{\partial \mathbf{v}}{\partial t}+(\mathbf{v} \cdot \nabla) \mathbf{v}=-\frac{\nabla P}{\rho}+v \nabla^{2} \mathbf{v}+\frac{\rho_{e} \mathbf{E}}{\rho}
$$


where $\mathbf{v}$ is the fluid velocity, $P$ the pressure, $\rho$ the mass density, $v$ the kinematic viscosity, $\rho_{e}$ the charge density, and $\mathbf{E}$ the electric field. The charge density $\rho_{e}$ and current density $\mathbf{j}$ include contributions due to all charged species present. These obey the equations of charge conservation:

$$
\nabla \cdot \mathbf{j}_{i}+\frac{\partial \rho_{i}}{\partial t}=R\left(\left\{n_{i}\right\}\right)
$$

where

$$
\begin{gathered}
\rho_{e}=\sum_{i} \rho_{i}=\sum_{i} n_{i} q_{i} \\
\mathbf{j}=\sum_{i} \mathbf{j}_{i}=\sum_{i} n_{i} \mu_{i}\left|q_{i}\right| \mathbf{E}-q_{i} D_{i} \nabla n_{i}+n_{i} q_{i} \mathbf{v}
\end{gathered}
$$

The subscript $i$ refers to the $i$ th charge species, and $n_{i}, \mu_{i}, q_{i}$, and $D_{i}$ are the number density of the charged species, its mobility, ionic charge, and diffusion constant, respectively. We also have the equations of electrostatics:

$$
\begin{aligned}
\nabla \cdot \varepsilon \mathbf{E} & =\rho_{e} \\
\nabla \times \mathbf{E} & =0
\end{aligned}
$$

where $\varepsilon$ is the permittivity. Normally, one can assume that the fluid is incompressible, so

$$
\nabla \cdot \mathbf{v}=0
$$

In addition, we require equations specifying $R\left(\left\{n_{i}\right\}\right)$, which is a source term governing the generation and recombination of the various charged species and which in general will depend on the concentrations of all species. These additional equations may include, for example, rate equations for any electrochemical reactions. The system of equations (1)-(7) must be solved subject to the appropriate boundary conditions, which would include boundary conditions on the velocity, the charge distribution, and the field.

Liquid crystals are weak electrolytes whose conductivity is due to ionic impurities. ${ }^{(7)}$ Under the influence of an electric field, these ions move through the fluid with a velocity determined by a mobility $\mu$ which is in general a tensor quantity. Typically $\mu_{\perp} \approx \mu_{\|} \approx 10^{-10} \mathrm{~m}^{2} / \mathrm{V}$ sec. Ions may be created and destroyed by dissociation/recombination reactions in the bulk or by electrode reactions. It is the usual practice to assign the liquid crystal a conductivity $\sigma$ given by

$$
\sigma=\sum_{i} n_{i} \mu_{i}\left|q_{i}\right|
$$


which is treated as a constant material parameter. This is an approximation which is only justified when the concentrations $n_{i}$ are field independent, constant in time and space, and when electrochemical effects are negligible. These conditions break down near electrodes or in screening layers near free surfaces, and also at very low frequencies where there is time for the ions to drift across the sample to the electrodes. ${ }^{(7)}$ Since all of these effects are present in our experiments, the conductivity is not a good material parameter in our case, and in Eq. (7) we must express the current in terms of the charge densities and mobilities explicitly.

There are similarities between this problem and that of RayleighBénard convection, where the driving force is due to the action of gravity on the dense fluid near the top of a fluid layer heated from below. ${ }^{(1,2)}$ In the case of electroconvection, however, the situation is complicated by fact that the spatially-varying charge distribution $\rho_{e}$ is itself a function of the field $\mathbf{E}$, so the body force term in the Navier-Stokes equation is nonlinear.

Various solutions to this system of equations have been carried out for the case of a DC field and, typically, a parallel plate capacitor geometry. ${ }^{(23.27)}$ None of these can be directly applied to our experiment because of the different boundary conditions and electric field configurations. For the case of injection of charges of one species from one electrode, these solutions give a critical voltage for the DC instability which depends only on material parameters. The injection process is usually modeled by setting $R\left(\left\{n_{i}\right\}\right) \equiv 0$ in Eq. (2) and imposing a boundary condition, assumed to be field-independent, on the charge density $n$. The actual magnitude of the critical voltage is strongly dependent on the assumptions made regarding the injection process; calculated values varying by a factor of at least 4 have been reported, all of which seem to be higher than experimental values. ${ }^{(7)}$

The theory of electroconvection has been studied in much detail in nematic liquid crystals in the case where the mechanism driving the instability involves the anisotropy of the material. ${ }^{(4-9)}$ In this case, terms must be included which describe the coupling of the flow to the director and hence to the axes of the tensor material parameters. These are not necessary in our case because our flow does not couple to the director. Flow is confined to the plane of layers within which the smectic A is isotropic.

We make no attempt to solve Eqs. (1)-(7) here, although work to that end is in progress. Rather, we will attempt to develop a qualitative picture of the processes occurring in our system by comparing results obtained in the contact and noncontact holders. Consider first the noncontact case. Here there is no injection of charges from the electrodes into the film. In the absence of injection there can be no instability at DC. ${ }^{(7)}$ Whatever 
charges already exist in the film will assume an equilibrium screening configuration such that the energy of the system is minimized, and no further changes will occur. This is in accord with our observation that the critical voltage for the onset of flow diverges at DC in the noncontact holder. The AC flow in the noncontact holder also cannot involve injection; it occurs because the charge configuration is thrown out of equilibrium when the applied field is rapidly changed. The charges then redistribute themselves toward a new equilibrium configuration assisted by flow if the rate of change of the field is large enough. With a sinusoidal applied field, $d \mathbf{E} / d t$ is proportional to $f$ and so $V_{c}^{\text {sine }}$ diverges as $f \rightarrow 0$. With square wave driving, $d \mathbf{E} / d t$ is limited by the slew rate of our function generator/ amplifier system and so $V_{c}^{\text {square }}$ approaches a constant at low frequency. At higher frequencies $V_{c}$ increases because the field is changing too rapidly for the charges in the film to respond. The behavior of the film in the noncontact holder is relatively straightforward and serves mainly to illustrate that in this geometry an instability is possible under $\mathrm{AC}$ conditions, even without injection.

In the contact holder, sustained flow is observed at DC. This flow must be driven by injection, by the reasoning given above. It is reasonable to assume that injection is also present to some degree at low but nonzero frequencies. Since the electrochemical processes which generate charges at the electrodes are slow, injection is not expected to be important at frequencies higher than a few Hz. ${ }^{(7)}$ The injected charges act to destabilize the equilibrium charge distribution near the electrodes. ${ }^{(21)}$ In our case $\mathrm{TCNQ}^{-}$ ions are created near the cathode. ${ }^{(31)}$ If the ions are injected faster than they can drift or diffuse away, they will pile up near the cathode. The charge distribution there will then contain more negative charge than when in equilibrium, and there will therefore be an excess force due to the field acting on these charges. If the force is large enough to overcome viscous effects, it will drive the film into motion.

This mechanism is usually named after Felici, ${ }^{(21)}$ and is well known in the study of hydrodynamic instabilities in insulating liquids, i.e., those in which all charges come from the injection process. ${ }^{(21-26)}$ In our films, however, not all the charges are due to injection, as demonstrated by the behavior of the noncontact holder. Thus, while the qualitative features of the Felici mechanism remain valid for our films, the detailed theory ${ }^{(23-25)}$ must be modified. Attempts to generalize the theory to include the effects of injection at both electrodes and of ion recombination have been carried out. $^{(25,27)}$ Turnbull ${ }^{(27)}$ allowed for a nonuniform space charge distribution of both signs of charge, and included the effects of generation and recombination of carriers in the bulk fluid. In a parallel plate geometry, he found an instability leading to small convection rolls close to the electrodes. The size 
of the rolls was of the order of the Debye length, $L_{\mathrm{D}}=(\varepsilon D / 4 \pi \sigma)^{1 / 2}$, where $\varepsilon$ is the dielectric constant, $D$ the diffusion constant, and $\sigma$ the conductivity, since in this geometry all the charges are concentrated in a region of size $\approx L_{\mathrm{D}}$ near the electrodes. The geometry of the field and charge distributions is considerably more complicated in the case of the film, so this calculation cannot be directly applied. However, we believe that the basic picture is correct, at least in the DC case. Very little work has been done toward extending these calculations to the case of nonzero frequency. ${ }^{(7,29,30)}$

These has been some previous experimental work on electroconvection in thin films of nematic and isotropic liquid crystals by Faetti et al ${ }^{(18-20)}$ We now discuss the similarities and differences between our results and theirs. Faetti et al. ${ }^{(18-20)}$ performed measurements on suspended films of MBBA ( $N$ - $p$-methoxybenzilidene- $p$ - $n$-butylaniline), a material which has $\Delta \varepsilon<0$ in the nematic phase. They worked with films of at least $5 \mu \mathrm{m}$ thickness, roughly an order of magnitude thicker than our films. In both the nematic and isotropic phases they observed an instability leading to a vortexlike flow pattern above a critical voltage. In nematic films thicker than $7 \mu \mathrm{m}$, this instability was preceded by a bifurcation to a different state, their "domain mode," which they attribute to the Carr-Helfrich mechanism. ${ }^{(8,16)}$ We did not observe this state in our thinner smectic A films. Also, they observed that the director was reoriented by the flow in the nematic phase; we find no reorientation in the smectic A film.

Their system differed from ours because neither the nematic nor isotropic phases have the layered structure of the smectic, so their films were of nonuniform thickness at rest, with the shape determined by surface tension. The free surfaces of their films were strongly distorted and the thickness modified by the flow. The planform of the vortices they observed was tilted with respect to the axes of the film holder, unlike ours. They followed the instability up to frequencies of order $100 \mathrm{~Hz}$, and report that the vortices remained the same size at all frequencies, whereas ours get smaller above a few $\mathrm{Hz}$. They found that the onset voltage was linear in the film thickness, as we also observed. The DC wavelength of the vortex pattern appears to be the same as observed by us: we found $\lambda / d=$ $1.30 \pm 0.05$, Faetti et al. measured $1.4 \pm 0.3$ for the same quantity. ${ }^{(20)}$

It seems clear that the instability studied by Faetti et al. is closely related to ours, but it differs in many important respects. It is difficult to know how many of the differences can be attributed to their larger film thickness and to thickness nonuniformity. In general, it seems that the uniform thickness of the smectic film makes this system more controllable and reproducible.

Faetti et al. ${ }^{(20)}$ propose a model for their "vortex mode" instability 
based on the interaction of charges on the free surfaces of the film interacting with the applied field. These surface charges are assumed to be in screening layers whose thickness is on the order of the Debye length $L_{\mathrm{D}}$, defined above. For both their films and ours $L_{\mathrm{D}}$ is less than about $0.1 \mu \mathrm{m},{ }^{(7)}$ which in their case is much less than their film thickness $s$. They incorporate the fact that $s \gg L_{\mathrm{D}}$ into their theory by treating the charges, currents, and forces as purely surface terms which in turn drive the uncharged interior of the film. This treatment is not satisfactory for our smectic films, however, because in our case $L_{\mathrm{D}} \approx s$ and the concept of a surface layer is not very well defined.

\section{CONCLUSION}

We have presented results of a study of convective flow near its onset in a freely suspended film of smectic A liquid crystal. This system behaves like a two-dimensional isotropic liquid. The flow displays two distinct patterns at the onset. Large vortices which span the width of the film are observed at low frequencies; these give way to lines of smaller vortices near each electrode at higher frequencies. We have studied the behavior of the critical voltage and the wavelength of the pattern as functions of the frequency of the applied field and the geometry of the film. It is clear that much theoretical work is required before a quantitative understanding of all of the observations will be possible. The driving mechanism involves several aspects of the general problem of electroconvection which are poorly understood at present. However, the simple flow behavior of the film makes it an attractive system in which to study the formation and evolution of one-dimensional patterns.

\section{REFERENCES}

1. G. Ahlers, in Lectures in the Sciences of Complexity, D. Stein, ed. (Addison-Wesley, Reading, Massachusetts, 1989).

2. F. Busse, in Hydrodynamic Instabilities and the Onset of Turbulence, H. L. Swinney and J. P. Gollub, eds. (Springer, Berlin, 1984).

3. R. C. DiPrima and H. L. Swinney, in Hydrodynamic Instabilities and the Onset of Turbulence, H. L. Swinney and J. P. Gollub, eds. (Springer, Berlin, 1984).

4. I. Rehberg, B. Winkler, M. de la Torre Juarez, S. Rasenat, and W. Schöpf, in Festkörperprobleme/Advances in Solid State Physics, U. Rössler, ed. (Vieweg, Braunschweig, 1989).

5. E. Dubois-Violette, P. G. de Gennes, and O. Parodi, J. Phys. (Paris) 32:305 (1971).

6. P. G. de Gennes, The Physics of Liquid Crystals (Clarendon Press, Oxford, 1979).

7. L. M. Blinov, Electro-Optical and Magneto-Optical Properties of Liquid Crystals (Wiley, New York, 1983).

8. W. Helfrich, J. Chem. Phys. 51:4092 (1969). 
9. E. Bodenschatz, W. Zimmermann, and L. Kramer, J. Phys. (Paris) 49:1875 (1988).

10. S. W. Morris, J. R. de Bruyn, and A. D. May, in Nonlinear Evolution of Spatio-Temporal Structures in Dissipative Continuous Systems, F. Busse and L. Kramer, eds. (Plenum Press, New York, 1990).

11. S. W. Morris, J. R. de Bruyn, and A. D. May, Phys. Rev. Lett. 65:2378 (1990).

12. A. J. Leadbetter, J. C. Frost, J. P. Gaughan, G. W. Gray, and A. Mosly, J. Phys. (Paris) 40:375 (1979).

13. L. Léger and A. Martinet, J. Phys. (Paris) 37:C3-89 (1976).

14. R. G. Horn and M. Kléman, Ann. Phys. 3:229 (1978).

15. G. Friedel, Ann. Phys. (Paris) 18:273 (1922).

16. R. Pindak and D. Moncton, Phys. Today 35(5):57 (1982).

17. D. A. Dunmur, M. R. Manterfield, W. H. Miller, and J. K. Dunleavy, Mol. Cryst. Liq. Cryst. 46:127 (1978).

18. S. Faetti, L. Fronzoni, and P. A. Rolla, J. Phys. (Paris) 40:C3-497 (1979).

19. S. Faetti, L. Fronzoni, and P. A. Rolla, J. Chem. Phys. 79:1427 (1983).

20. S. Faetti, L. Fronzoni, and P. A. Rolla, J. Chem. Phys. 79:5054 (1983).

21. N. Felici, Rev. Gen. Elect. 78:717 (1969); J. Phys. (Paris) 37:C1-117 (1976).

22. J. M. Schnieder and P. K. Watson, Phys. Fluids 18:1948 (1970).

23. P. Atten and R. Moreau, J. Mécanique 11:471 (1972).

24. J. C. Lacroix, P. Atten, and E. J. Hopfinger, J. Fluid Mech. 69:539 (1975).

25. J. C. Lacroix and P. Atten, C. R. Acad. Sci. 278A:689 (1974); J. Electrostatics 5:453 (1978).

26. P. Atten and J. C. Lacroix, J. Mécanique 18:469 (1979); J. Electrostatics 5:439 (1978).

27. R. J. Turnbull, J. Phys. D 6:1746 (1973).

28. R. Bruinsma and S. Alexander, J. Chem. Phys. 92:3074 (1990).

29. P. Atten, B. Malraison, and S. Ali Kani, J. Electrostatics 12:477 (1982).

30. M. I. Barnik, L. M. Blinov, S. A. Pikin, and A. N. Trufanov, Sov. Phys. JETP 45:396 (1977).

31. R. Cocco, F. Gaspard, and R. Herino, J. Chim. Phys. 76:383 (1979).

32. R. Foster, Organic Charge Transfer Complexes (Academic Press, New York, 1969).

33. M. Born and E. Wolf, Principles of Optics (Pergamon Press, New York, 1980).

34. C. Rosenblatt and N. Amer, Appl. Phys. Lett. 36:432 (1980). 